\title{
ESTABLECIENDO PROTOCOLO DE APOYO EN MICROCIRUGÍA*
}

\author{
Drs. Marilú Sylvester F. ${ }^{1}$, Héctor Roco M. ${ }^{2}$ \\ ${ }^{1}$ Cirugía Plástica del Hospital Clínico de la Fuerza Aérea de Chile. \\ Santiago, Chile.
}

\begin{abstract}
\section{Developing a protocol to support microsurgery}

Microsurgery is a developing technique in our setting, and it's success can be related to elements that are not related to the surgeon. Because of this, the Plastic Surgery Team in the Chilean Airforce Hospital has become aware of the need to develop a support protocol for microsurgery, which can be used in any setting throughout our country. It's focus is set in optimizing and controlling physiological and anesthetic variables, and those not related to the technique itself, which can influence the microsurgery outcome and the patients perioperative morbi-mortality.
\end{abstract}

Key words: Microsurgery, free flap surgery, complication, perioperative care, flap monitoring.

\section{Resumen}

La microcirugía es una técnica en pleno desarrollo en nuestro medio y su éxito puede estar condicionado a veces a elementos externos al cirujano. Es por esto que, en el Hospital de la Fuerza Aérea de Chile, el Equipo de Cirugía Plástica ha notado la necesidad de diseñar un protocolo de apoyo a la microcirugía, el cual sea posible implementar en cualquier medio nacional. Su enfoque está dirigido a optimizar y controlar las variables fisiológicas, anestésicas y externas a la técnica quirúrgica en si misma, que podrían incidir en el éxito de la microcirugía como en la morbimortalidad perioperatoria de los pacientes.

Palabras clave: Microcirugía, colgajo libre, cuidados perioperatorios, monitorización colgajo libre, complicaciones.

\section{Introducción}

Los colgajos microquirúrgicos nacen como una alternativa frente a la necesidad de realizar una reconstrucción de calidad y de minimizar las secuelas de la zona dadora. Sin embargo, la falla de la microcirugía puede implicar una gran carga física y emocional para los pacientes, así como también podría definir el pronóstico de ellos. En la literatura, la falla microvascular varía entre un $5-10 \%$ para los colgajos libres y entre un $15-30 \%$ para los reimplantes ${ }^{1}$. Se ha evidenciado que existe una

*Recibido el 2 de septiembre de 2014 y aceptado para publicación el 17 de diciembre de 2014.

Los autores no refieren conflictos de interés.

Correspondencia: Marilú Sylvester F.

Marilu.sylvester@gmail.com 
tasa mayor de fallas en colgajos realizados en sitio de trauma ${ }^{2}$ que en otras cirugías $(15 \%$ vs $5 \%)$. Así mismo se ha comunicado que el $80 \%$ de las fallas de las anastomosis ocurren en las primeras $48 \mathrm{~h}^{3,4}$, y que la mayoría de las causas corresponderían a la trombosis de las anastomosis arterial o venosa ${ }^{5}$. También se ha descrito que el tipo de colgajo podría influir en la incidencia de falla, ya que, por ejemplo, podría tener una mayor dificultad en su monitorización (ej. colgajo muscular), o presentar una circulación más límite (ej. colgajo perforante). Esto ha quedado evidenciado en el estudio de Winterton ${ }^{6}$, donde encontró que los colgajos musculares vs fasciocutáneos tuvieron mayor incidencia de fracaso. Por otro lado, en el artículo de Casesahmet Demir ${ }^{7}$, los colgajos de perforantes vs axiales presentaron mayor tasa de falla y menor éxito en el rescate. Sin embargo, es ampliamente reconocido que aún habiendo una elección y elevación correcta del colgajo, en conjunto con una anastomosis técnicamente bien realizada, el procedimiento podría fracasar por factores locales, sistémicos internos y/o externos; o por complicaciones médico-quirúrgicas del paciente, incluyendo la muerte.

Es por esto que en nuestro hospital, el Equipo de Cirugía Plástica se planteó como objetivo el diseño de un protocolo a cumplir en el periodo intra y perioperatorio enfocado principalmente en el manejo de las variables fisiológicas, anestésicas y externas a la técnica quirúrgica en si misma. Creemos que su aplicación podría ayudar a evitar o disminuir complicaciones médico-quirúrgicas del paciente, así como también contribuir al éxito de la microcirugía.

\section{Elaboración del protocolo}

Para el diseño del protocolo dos cirujanos plásticos con entrenamiento microquirúrgico del hospital plantearon las variables preoperatorias, intraoperatorias y postoperatorias médicas/quirúrgicas relevantes a controlar y/o monitorizar. Lo anterior se contrapuso y se complementó con protocolos disponibles en la literatura, adaptándolo a la realidad intrahospitalaria nacional.

\section{Variables}

Entre las variables definidas como importantes, algunas correspondieron a factores relacionados al paciente, como son la edad, sus comorbilidades y hábitos; mientras que otras variables se correspondieron a parámetros fisiológicos, medidas de prevención de complicaciones (trombosis venosa profunda, úlceras por presión, infección, úlceras por estrés) y la evaluación constante de la vitalidad del colgajo microquirúrgico en el postoperatorio (Tabla 1).

\section{Edad y comorbilidades}

Si bien la microcirugía es una técnica ya bien establecida, ésta ha generado ciertas aprehensiones para su aplicación en la población pediátrica y en la de adultos mayores. En la primera, por el menor calibre de los vasos para realizar la microanastomosis y su labilidad fisiológica-hemodinámica, y en la segunda debido a la preexistencia de comorbilidades y mayor deterioro del estado general. Sin embargo, varios artículos publicados han respaldado esta técnica en ambos extremos de la vida ${ }^{8-11}$. Aunque en algunos estudios la población de mayor edad (sobre 70 años $)^{12}$ ha presentado una mayor tasa de complicaciones médicas e incluso mortalidad, lo disponible en la literatura apuntó a que el factor de riesgo para mayores complicaciones, más que la edad, era la clasificación de $\mathrm{ASA}^{3}$. Por lo anterior, se incluyó dentro de las consideraciones preoperatorias del protocolo, la identificación de las comorbilidades del paciente y la evaluación por un equipo multidisciplinario (anestesista, médico internista, nutricionista, etc.). Para la evaluación del estado basal del paciente se consideraron exámenes generales que objetivaran, entre otros, el estado nutricional (desnutrición, obesidad), función renal, posible grado de anemia o alteración de la coagulación. Ciertos artículos correlacionaron algunos factores de riesgo con mayor tasa de falla en microcirugía, como una creatinina sobre $1,28 \mathrm{mg} / \mathrm{dl}$, la presencia de arteriosclerosis ${ }^{13}$ y el uso de broncodilatadores ${ }^{14}$. Sin embargo, las

Tabla 1. Variables incluidas en el protocolo

\section{Variables definidas}

1. Edad

2. Comorbilidades: diabetes, enfermedad ateroesclerótica, enfermedad cardíaca, enfermedad pulmonar, enfermedad renal, enfermedad hepática, hipertensión arterial, dependencia a drogas, estado nutricional, radioterapia, quimioterapia, etc.

3. Hábitos del paciente: alcohol, tabaco, drogas.

4. Variables fisiológicas: temperatura, volemia, presión arterial, frecuencia cardíaca, saturación de oxígeno, diuresis, hematocrito/hemoglobina, estado ácido-base.

5. Profilaxis de trombosis venosa profunda (TVP)/tromboembolismo pulmonar (TEP).

6. Profilaxis antibiótica.

7. Profilaxis de úlceras por estrés.

8. Prevención de úlceras por presión.

9. Monitorización intra operatoria y postoperatoria del colgajo microquirúrgico. 
comorbilidades determinaron mayor incidencia de complicaciones médicas que quirúrgicas ${ }^{4}$.

Diversos estudios han demostrado que la estabilización preoperatoria de comorbilidades como la diabetes, hipertensión arterial, insuficiencia renal, enfermedad pulmonar, etc., disminuyó la morbilidad perioperatoria e incluso la mortalidad de los pacientes. Se ha sugerido enfatizar el estudio en enfermos con sospecha de patología cerebrovascular con arteriosclerosis avanzada, ya que se han asociado a un riesgo mayor de infartos cardíacos y eventos cerebrovasculares. Por otro lado, en pacientes con enfermedades cardiovasculares, la administración de atenolol en el pre y postoperatorio disminuyó la morbimortalidad $^{15}$. En cuanto a la diabetes mellitus, concordamos que debe intentarse un buen control metabólico de la glicemia, ya que esto ha reducido la morbimortalidad perioperatoria en diversos estudios. También se ha descrito que la hiperglicemia se correlaciona con mayor permeabilidad vascular y consecuentemente mayor edema de los tejidos, aumentando así la presión extravascular en el colgajo, lo que afecta negativamente la perfusión de éste ${ }^{12,15}$.

Otros factores a monitorizar dentro de lo considerado por los autores y descrito en otros protocolos, fueron el uso del tabaco y la dependencia a sustancias. La suspensión del tabaco entre 2 a 4 semanas antes de la cirugía es una medida transversal a todo acto quirúrgico y se ha asociado a menor morbilidad broncopulmonar y, en algunos casos, a mejor cicatrización de la zona donante; sin embargo, su consumo no se ha correlacionado con una mayor tasa de falla en la microanastomosis ${ }^{12,14}$. La evaluación y objetivación de la dependencia al alcohol u otras drogas ha sido otro punto a destacar, ya que el síndrome de privación podría llevar a la falla de la cirugía, en parte por un estado hiperadrenérgico que lleva a la vasoconstricción y por otra parte por la agitación psicomotora del paciente. En pacientes lábiles y cirugía de larga duración planteamos considerar la realización de la microcirugía en dos etapas: levantar el colgajo y preparar los vasos receptores en un primer tiempo, y realizar las microanastomosis en un segundo tiempo. Esto con el fin de disminuir la duración de la cirugía sobre $10 \mathrm{~h}$, lo que se ha correlacionado con el aumento de la tasa de falla del colgajo y de la morbimortalidad en pacientes más lábiles y/o de edad avanzada ${ }^{3,14}$. Hemos encontrado evidencia contradictoria respecto a la radioterapia en la zona de los vasos receptores, ya que según algunos autores podría perjudicar la cirugía ${ }^{14}$, mientras que para otros no tendría repercusión ${ }^{3}$. Se sugiere considerar diferir la microcirugía si es posible después de los 12 meses, por ejemplo en la reconstrucción mamaria, ya que se ha visto una mayor seguridad en la anastomosis $^{16}$. En los pacientes que han sido tratados con quimioterapia se ha recomendado tener un recuento adecuado de leucocitos sobre $3.000 / \mathrm{mm}^{3}$, el cual se recupera habitualmente después de la $3^{\mathrm{a}}$ semana postquimioterapia ${ }^{17}$, con el fin de asegurar una adecuada cicatrización y menor riesgo de infección (Tabla 2).

\section{Profilaxis de tromboembolismo, antibiótica, de úlceras por estrés y de úlceras por presión}

Como la microcirugía es un procedimiento de moderado-alto riesgo de fenómenos tromboembólicos se han descrito protocolos de profilaxis de TVP para cirugía de alto riesgo (cirugía prolongada, trauma, cáncer, etc.) con heparina en dosis profiláctica y compresión neumática de las extremidades. La literatura disponible ha evidenciado que no ha habido mayor incidencia de hematoma o sangrado postoperatorio con el uso de heparina tanto en profilaxis, como en irrigación tópica o como en bolo en el intraoperatorio ${ }^{1,15}$. Sin embargo, su uso como anticoagulación ha aumentado las complicaciones por sangrado y por hematoma, y no se ha evidenciado que disminuya la falla por trombosis de la microanastomosis ${ }^{1,18}$. Otro fármaco que ha sido usado frecuentemente es la aspirina, con una dosis recomendada de $100 \mathrm{mg}$ al día vía oral y no mayor. Ésta, en dosis bajas, inhibe la ciclooxigenasa plaquetaria, la cual produce tromboxano, y genera una mínima inhibición de la producción de prostaciclina derivada del endotelio ${ }^{1}$. La aspirina en combinación con heparina de bajo peso molecular (HBPM) como profilaxis ha podido usarse de forma segura para el paciente, pero sin evidencia clara que haya mostrado beneficios en la prevención o disminución de falla de la microanastomosis $3,4,19,20$.

La profilaxis antibiótica debe efectuarse según protocolos ya establecidos en cirugía general, media hora antes de la incisión, y repetirse según el tiempo operatorio, pérdida de sangre y el uso de isquemia ${ }^{15}$. La revisión de la literatura por $\mathrm{Kruse}^{3}$, realizada en microcirugías de cabeza y cuello, apoya la profilaxis de antibióticos de 3 dosis hasta 5 días, siendo en este caso la combinación de amoxicilina y ac. clavulánico lo de elección. Para las cirugías de tejidos blandos sin entrar en cavidades, la profilaxis que hemos recomendado es el uso de cefazolina, o clindamicina en pacientes alérgicos. En el caso de fracturas expuestas $\mathrm{u}$ otras lesiones que pudiesen estar contaminadas o infectadas, la administración del antibiótico debe ser según lo encontrado en el cultivo intraoperatorio, correspondiendo a un tratamiento más que a profilaxis.

En todo paciente que es sometido a cirugías de larga duración, que recibe la administración de múltiples fármacos, muchas veces asociado a trauma o portador de múltiples patologías, se recomienda la profilaxis de úlceras por estrés, siendo lo habitual el 


\section{Tabla 2. Variables preoperatorias a controlar}

\section{Comorbilidades}

a. Evaluación con equipo multidisciplinario y estabilización de patologías de base

i. ASA del paciente y compensación de comorbilidades: hipertensión arterial, insuficiencia renal, diabetes, enfermedad broncopulmonar, etc.

1. Riesgo coronario: Atenolol $5 \mathrm{mg} 30 \mathrm{~min}$ antes de cirugía, en el postoperatorio inmediato y durante la hospitalización

2. Diabéticos: mantener glicemias entre 80 y $110 \mathrm{mg} / \mathrm{dl}$

3. Enfermos con patología broncopulmonar: optimizar con broncodilatadores y apoyo kinesiológico previo a la cirugía

4. Evaluar en pacientes lábiles procedimiento en dos tiempos para evitar cirugías prolongadas (mayor a $10 \mathrm{~h}$ )

5. Radioterapia: Si los vasos receptores han recibido radioterapia, si es factible, diferir microcirugía 12 meses posterior a la radiación

6. Exámenes preoperatorios: hemograma, pruebas de coagulación, función renal, balance hidroelectrolítico y otros según patología específica

a. Evaluación nutricional, obesidad-desnutrición

i. Albúmina sobre $3 \mathrm{~g} / \mathrm{dl}$, prealbúmina cercana a rangos normales

b. Tratamiento de anemia previo a cirugía

c. Recuento de leucocitos sobre $3.000 / \mathrm{mm}^{3}$

\section{Hábitos}

a. Tabaco: cese 2-4 semanas previo a cirugía y posterior a ésta

b. Dependencias a sustancias: evaluación y tratamiento para evitar síndrome de privación

i. alcohol, nicotina, benzodiacepinas, etc.

3. Manejo de volemia

a. Corregir hipovolemia precirugía en los casos de trauma

i. Evitar la sobrehidratación

ii. Ajustar aportes según diuresis: $0,5-1 \mathrm{ml} / \mathrm{k} / \mathrm{h}$

4. Prevención de hipotermia

a. Mantener temperatura ambiente cercana a $24^{\circ} \mathrm{C}$

b. Manta calefactora $1 \mathrm{~h}$ antes de inducción, evitar sudoración

5. Tromboprofilaxis

a. Comenzar compresión neumática antes de la inducción anestésica y continuar en el intra y post operatorio

b. Iniciar heparina de bajo peso molecular 6 a $12 \mathrm{~h}$ post cirugía

6. Antibioprofilaxis

a. Administración del antibiótico 30 min antes de inducción

i. Considerar:

1. Vida media del antibiótico

2. Duración del procedimiento (mayor a $5 \mathrm{~h}$ )

3. Pérdida de sangre mayor a $1.500 \mathrm{ml}$

4. Uso de torniquete

b. Tipo de herida:

i. Microcirugía limpia

1. Cefalosporina primera generación o Clindamicina si existe alergia a la primera

ii. Microcirugía de cabeza y cuello

1. Amoxicilina-Ac. Clavulánico 
uso de omeprazol o similares. En cuanto a la prevención de las úlceras por presión, se ha propuesto que su manejo se inicie desde el preoperatorio (mesa quirúrgica acondicionada, protecciones para prominencias óseas, etc.). La monitorización y prevención activa en el intra y postoperatorio, implicando el cambio de posición, uso de colchón antiescaras, apoyo de kinesiología y deambulación precoz, son los pilares fundamentales para su prevención ${ }^{4,21,22}$ (Tablas 2, 3 y 4 ).

\section{Control de parámetros fisiológicos}

Tanto en el intraoperatorio como en el postoperatorio se ha determinado la importancia de la monitorización de parámetros fisiológicos como son: temperatura, presión arterial, frecuencia cardíaca, saturación de oxígeno, diuresis, hemoglobina y gases. Para ello se ha sugerido, aparte de la monitorización de rutina no invasiva intraoperatoria, el uso de medición de temperatura central y en algunos casos la medición de presión arterial invasiva (línea arterial); medidas más agresivas de monitorización han sido poco utilizadas por nuestro equipo al igual que lo descrito en la literatura ${ }^{21}$. El uso de vasoconstrictores aún se encuentra en debate; hay algunos estudios que han evidenciado que no aumentarían la pérdida de colgajos libres ${ }^{14,21}$, sin embargo se recomienda prescindir, en la medida de lo posible, del uso de estos fármacos. En el intraoperatorio o en el caso que el paciente se encuentre con ventilación mecánica

Tabla 3. Variables intraoperatorias a controlar

1. Manejo de Hemodinamia

a. Manejo de Volemia:

i. Balance restrictivo de fluido con balance neutro o positivo de 2 litros y volumen total de coloides máximo de 2 litros

a. Mantener presiones arteriales sistólicas sobre $100 \mathrm{mmHg}$

b. Reemplazar pérdidas insensibles con suero fisiológico isotónico tibio

c. Reemplazar pérdida de sangre con coloides tibios y hemoderivados

i. Administración de coloides: sobre $20-30 \mathrm{ml} / \mathrm{kg} / 24 \mathrm{~h}$ pueden aumentar la morbilidad perioperatoria

ii. Balance hídrico: diuresis $>0,5-1 \mathrm{ml} / \mathrm{kg} / \mathrm{h}$

iii. Evitar el uso de vasoconstrictores

2. Manejo del estado ácido/base

a. Evitar hiperventilación e hipoventilación

b. Control intraoperatorio gases arteriales/venosos

3. Prevención de hipotermia

a. Mantener delta de temperatura central/periférica menor a $1^{\circ} \mathrm{C}$

b. Manejar temperatura sobre $36,5^{\circ} \mathrm{C}$

i. Manta calentadora

ii. Temperatura ambiente de sala a $24^{\circ} \mathrm{C}$

iii. Reposición de volumen con soluciones tibias

iv. Minimizar tiempos de reposicionamiento y operatorios

4. Tromboprofilaxis

a. Compresión neumática

5. Prevención de úlceras por presión

a. Protección de puntos de apoyo, colchón antiescaras, etc.

b. Protección ocular

c. Movilización intraoperatoria

i. Evitar lesiones por mala posición (plexo braquial por ej.)

6. Posición de drenaje venoso del colgajo y curación no compresiva

7. Despertar al paciente sin agitación ni dolor 
Tabla 4. Variables postoperatorias a controlar

1. Optimización de perfusión del colgajo:

a. Volemia adecuada:

i. Reemplazar pérdidas insensibles con suero fisiológico isotónico tibio

ii. Reemplazar pérdida de sangre con coloides tibios y hemoderivados

1. Buscar mantener hemoglobina alrededor de $10 \mathrm{~g} / \mathrm{dl}$ y hematocrito alrededor de $30 \%$

2. La administración de coloides sobre $20-30 \mathrm{ml} / \mathrm{kg} / 24$ puede aumentar la morbilidad perioperatoria

iii. Balance hídrico: obtener débito urinario sobre $0,5-1 \mathrm{ml} / \mathrm{kg} / \mathrm{h}$

iv. Evitar el uso de vasoconstrictores

b. Oximetría de Pulso: Saturación $>95 \%$

c. Prevención de Hipotermia

i. Manta calentadora

ii. Temperatura ambiente de sala $24^{\circ} \mathrm{C}$

d. Sedación post cirugía primeras horas
i. Control del dolor
ii. Evitar movimientos excesivos/calosfríos

e. Prevención trombosis microanastomosis

i. Aspirina $100 \mathrm{mg} /$ día vo por 30 días

ii. Heparina bajo peso molecular profiláctica por 14 días

f. Control de factores locales externos:
i. Curación apropiada (no compresiva)
ii. Evitar presión externa sobre el colgajo
iii. Mantener posición de drenaje venoso

2. Profilaxis de úlceras por estrés

3. Tromboprofilaxis

a. Sistema compresivo neumático: suspender al deambular

b. Heparina bajo peso molecular profiláctica

c. Primeras $24 \mathrm{~h}$ reposo absoluto y segundo día puede movilizarse a silla

i. En colgajos en extremidades inferiores, la pierna debe estar en posición de drenaje venoso y levantar al paciente con venda elasticada

4. Apoyo de kinesiología

a. Considerar movilización temprana para evitar eventos tromboembólicos, úlceras por presión, complicaciones respiratorias, etc.

5. Monitorización del colgajo

a. Vigilancia con Doppler: cada 60 min primer día, cada 2 h segundo día, cada 4 h al tercer y cuarto día, y cada 4 a 8 $\mathrm{h}$ hasta el alta

b. Temperatura del colgajo al tacto

c. Llene capilar: normal entre 2 ó 3 segundos, realizar en forma horaria primer y segundo día

i. Menor a 2 segundos: congestión venosa

ii. Mayor a 3 segundos: falla en la irrigación arterial

iii. Se puede objetivar con pinprick test el estado del colgajo:

1. Punción con aguja y observar:

a. Coloración del sangrado oscura indica congestión venosa

b. Ausencia de sangrado indica falla en la irrigación arterial

d. Coloración del colgajo: anormal si éste se encuentra cianótico (congestión venosa) o pálido (falla en la irrigación arterial)

e. Consistencia del colgajo: si se encuentra aumentada indica congestión, si se encuentra con pérdida del turgor indica falla en la irrigación; si se observa aumento de volumen localizado puede indicar hematoma 
en el postoperatorio, ha sido descrito que tanto la hiperventilación como la hipoventilación deben ser evitadas. La primera se ha asociado a alcalosis respiratoria, la cual puede conducir a una disminución del gasto cardiaco y con ello a vasoconstricción periférica. Por otra parte, se ha correlacionado el estado de hiperoxia a vasoconstricción y, por lo tanto, a una menor perfusión del colgajo. En el caso de la hipoventilación se ha asociado a una acidosis respiratoria, llevando a una reducción en la deformación de los eritrocitos y aumentando la liberación de catecolaminas, lo que podría influir en la microcirculación del colgajo ${ }^{22}$. Por lo anterior hemos recomendado en nuestro protocolo que el oxígeno administrado y la ventilación aplicada deben ajustarse cuidadosamente para mantener el estado ácido-base del paciente dentro de los parámetros fisiológicos.

La hipotermia (temperatura menor a $36,5^{\circ} \mathrm{C}$ ) ha sido descrita como un vasoconstrictor potente ${ }^{22}$, se ha asociado a coagulopatía, correlacionado a un mayor riesgo de infecciones de heridas operatorias, retardo en la cicatrización y agregación de eritrocitos y plaquetas. Su manejo y prevención debe comenzar en el preoperatorio con calefactor y temperatura adecuada en la sala de preanestesia, continuando en el intraoperatorio y en el postoperatorio.

Antes de realizar una microcirugía hay que considerar la volemia del paciente y tratar de buscar su optimización para lograr una adecuada presión de perfusión y evitar la vasoconstricción periférica. Es interesante considerar que los colgajos en el intraoperatorio y en el postoperatorio se encuentran en situaciones límites de irrigación, ya que se ha evidenciado que la circulación de éstos disminuye en las primeras $6-12 \mathrm{~h}$ hasta al $20 \%{ }^{22}$, explicable por un lado por la pérdida de inervación simpática y la subsecuente descarga de vasoconstrictores, y por otro lado por la disminución de presión de flujo, debido a la interrupción del aporte de otros vasos e injuria endotelial mediada por leucocitos. La hipovolemia se ha establecido como causa de vasoconstricción y, por consecuencia, de disminución de perfusión en el colgajo libre. Según algunos estudios la hipervolemia también se ha correlacionado con mayor riesgo de complicaciones médicas postoperatorias ${ }^{14}$. Así, la hemodilución extrema, por el uso excesivo de cristaloides, aumenta el edema del colgajo y determina un efecto procoagulante por desbalance de los factores de coagulación ${ }^{21,22}$. Respecto del manejo de la volemia, la monitorización de la diuresis es primordial, teniendo como objetivo un débito urinario entre 0,5 a $1 \mathrm{ml} / \mathrm{kg} / \mathrm{h}$. Las pérdidas insensibles deben ser reemplazadas con suero fisiológico isotónico tibio y las pérdidas de sangre con coloides tibios y/o sangre o sus derivados. Está descrito que los coloides que son administrados en un volumen mayor de
20-30 $\mathrm{ml} / \mathrm{kg} / 24 \mathrm{~h}$ podrían aumentar la morbilidad perioperatoria. En cuanto a parámetros hemáticos, el objetivo es mantener hematocrito entre $30-35 \%$ $\mathrm{y}$ hemoglobina alrededor de $10 \mathrm{~g} / \mathrm{dl}$. Con estos rangos se ha evidenciado una viscosidad en la sangre entre 3,5 y $4 \mathrm{cP}$. (fisiológico 4,5 cP.), con lo que se conservaría el aporte de oxígeno a los tejidos y se mantendría una viscosidad de la sangre reducida, disminuyendo así la posibilidad de trombosis de la microanastomosis ${ }^{22}$ (Tablas 3 y 4 ).

\section{Dolor y agitación}

El uso de analgesia y la mantención del paciente sin agitación son fundamentales en el momento de la extubación y durante el postoperatorio para evitar el dolor y/o la vasoconstricción secundaria a estados hiperadrenérgicos. Así como en pacientes sometidos a otras cirugías plásticas, en algunos pacientes microquirúrgicos hemos tenido una buena experiencia usando dexmetomidina intraoperatoria y como sedación postoperatoria para evitar calosfríos y mantener al paciente sin dolor ni agitación. Existe un estudio experimental en cerdos, que mostró que su uso favorecería la preservación de la microcirculación en el colgajo, pero no se han publicado estudios clínicos al respecto ${ }^{4,21,22}$ (Tablas 3 y 4 ).

\section{Manejo de variables externas locales y monitorización del colgajo}

Una vez finalizada la cirugía el equipo quirúrgico debe resguardar la posición de drenaje venoso y verificar ausencia de compresiones en el colgajo y/o su pedículo. En nuestro protocolo se estableció el monitoreo estricto del colgajo en el postoperatorio por enfermería o el personal de apoyo, sobre todo dentro de las primeras $48 \mathrm{~h}$, ya que el porcentaje de éxito del rescate en las primeras $24 \mathrm{~h}$ está descrito entre el $80 \%$ y el $100 \%$, mientras que entre el segundo al sexto día desciende a rangos entre $28 \%$ y $58 \%{ }^{3,5,7}$. Hemos elegido la monitorización del colgajo mediante la observación clínica y el Doppler manual, ya que su combinación nos ha permitido una detección precoz de alguna complicación en la perfusión del colgajo y, a la vez, han sido de fácil implementación por el personal de apoyo. El Doppler implantable está descrito como el método que muestra un nivel de evidencia mayor (2a) dentro de la literatura, a diferencia de otros métodos que hacen cuestionable su uso en clínica ${ }^{23}$; sin embargo, debido a los costos no se incluyó en nuestro protocolo (Tablas 3 y 4 ).

Una vez que se detecta compromiso de la circulación del colgajo se debe avisar al microcirujano. $\mathrm{Si}$ se estima necesario se puede descomprimir el pedículo, en caso de edema o hematoma, retirando algunos puntos del colgajo, y si éste se encuentra 


\section{Tabla 5. Rescate de Colgajo}

1. Tratamiento agresivo del hematoma o seroma bajo supervisión del microcirujano

a. Remover vendajes y suturas, optimizar retorno venoso (elevar zona del colgajo)

b. Protocolo de heparina subcutánea o sanguijuelas en los casos de congestión venosa y no poder revisar anastomosis o falla posterior al rescate

2. Revisión en quirófano por microcirujano

congestivo se puede aplicar gasas con heparina en los bordes. En la literatura se ha descrito un protocolo con heparina subcutánea para el rescate de colgajos congestivos, que creemos podría ser una mejor opción a las sanguijuelas, sin embargo, este método no está libre de complicaciones como hematoma, síncope por anemia y la necesidad de transfusiones (en la mayoría de los pacientes entre 5 a $12 \mathrm{U})^{24}$ (Tabla 5).

\section{Conclusión}

Creemos que este protocolo es un aporte ya que está enfocado a la evaluación y sistematización del manejo de las posibles variables independientes de la técnica microquirúrgica, las que podrían jugar un rol tanto en el éxito de la cirugía como en la morbimortalidad perioperatoria. Además este protocolo es posible de implementar en cualquier centro que realice microcirugía en nuestro medio.

\section{Referencias}

1. Matthew H. Conrad, William P. Adams Jr. Pharmacologic Optimization of Microsurgery in the New Millennium. Plast Reconstr Surg. 2001; 108:2088-96.

2. Egozi D, Fodor L, Ullmann Y. Salvage of compromised free flaps in trauma cases whit combined modalities. Microsurgery 2011;31:109-15.

3. Kruse A, Luebbers HT, Gra'Tz KW, Obwegeser JA. Factors Influencing Survival of Free-Flap in Reconstruction for Cancer of the Head and Neck: A Literature Review. Microsurgery 2010;30:242-8.

4. Gardiner MD, Nanchahal J. Strategies to ensure success of microvascular free tissue transfer. Journal of Plastic, Reconstructive \& Aesthetic Surgery 2010;63:e665ee673.

5. Smit JM, Acosta R, Zeebregts CJ, Liss AG, Anniko M, Hartman ED. Early reintervention of compromised free flaps improves success rate. Microsurgery 2007;27:612-6.

6. Winterton RI, Pinder RM, Morritt AN, Knight SL,
Batchelor AG, Liddington MI, et al. Long term study into surgical re-exploration of the free flap in difficulty. Journal of Plastic, Reconstructive \& Aesthetic Surgery 2010;63:1080e1086.

7. Demir A, Kucuker I, Keles MK, Demirtas Y. The effect of learning curve on flap selection, re-exploration, and salvage rates in free flaps; a retrospective analysis of 155 cases. Microsurgery 2013;33:519-26.

8. Lin CH, Mardini S, Wei FC, Lin YT, Chen CT. Free flap reconstruction of foot and ankle defects in pediatric patients: Long term outcome in 91 cases. Plast Reconstr Surg. 2006; 117:2478-87.

9. Rinker B, Valerio IL, Stewart DH, Pu LL, Vasconez HC. Microvascular free flap reconstruction in pediatric lower extremity trauma: A 10-year review. Plast Reconstr Surg. 2005;115:1618-24.

10. Ozkan O, Ozgentas HE, Islamoglu K, Boztug N, Bigat Z, Dikici MB. Experiences with microsurgical tissue transfers in elderly patients. Microsurgery 2005;25:3905.

11. Coskunfirat OK, Chen HC, Spanio S, Tang YB. The safety of microvascular free tissue transfer in the elderly population. Plast Reconstr Surg. 2005;115:771-5.

12. Chen HC, Coskunfirat OK, Ozkan O, Mardini S, Cigna E, Salgado CJ, et al. Guidelines for the optimization of microsurgery in atheroesclerotic patient. Microsurgery 2006;26:356-62.

13. Baek Kyu Kim, Yung Ki Lee, Keun Yung Park, Rong Min Baek, Chan Yeong Heo, Seok Chan Eun, et al. Analysis of multiple risk factors affecting the result of free flap transfer for necrotising soft tissue defects of the lower extremities in patients with type 2 diabetes mellitus. Journal of Plastic, Reconstructive \& Aesthetic Surgery 2014;67,:624e628.

14. Pattani KM, Byrne P, Boahene K, Richmon J. What Makes a Good Flap Go Bad?: A Critical Analysis of the Literature of Intraoperative Factors Related to Free Flap Failure. Laryngoscope 2010:120:717-23.

15. Evans BC, Evans GR. Microvascular Surgery. Plast Reconstr Surg. 2007;119:18e-30e.

16. Fosnot J, Fischer JP, Smartt JM, Low DW, Kovach SJ III, Wu LC, et al. Does Previous Chest Wall Irradiation Increase Vascular Complications in Free Autologous Breast Reconstruction? Plast Reconstr Surg. 2011;127:496-504.

17. Ariyan S, Kraft RL, Goldberg NH. An experimental model to determine the effects of adjuvant therapy on the incidence of postoperative wound infections II: evaluating preoperative chemotherapy. Plast Reconstr Surg 1990;65:338-45.

18. Chen CM, Ashjian P, Disa JJ, Cordeiro PG, Pusic AL, Mehrara BJ. Is the Use of Intraoperative Heparin Safe? Plast Reconstr Surg. 2008;121:49-53e.

19. Christopher J. Salgado CJ, Steven L. Moran SL, Mardini S. Flap Monitoring and Patient Management. Plast Reconst Surg. 2009;124:295e-299e. 
20. Brands MT, van den Bosch SC, Dieleman FJ, Bergé SJ, Merkx MA. Prevention of thrombosis after microvascular tissue transfer in the head and neck. A review of the literature and the state of affairs in Dutch Head and Neck Cancer Centers. Int J Oral Maxillofac Surg. 2010;39:101-6.

21. Gooneratne H, Lalabekyan B, Clarke S, Burdett E. Perioperative anaesthetic practice for head and neck free tissue transfer-a UK national survey. Acta Anaesthesiol Scand. 2013;57:1293-300.
22. Hagau N, Longrois D. Anesthesia for free vascularized Tissue Transfer. Microsurgery 2009;29:161-7.

23. Pratt GF, Rozen WM, Chubb D, Whitaker IS, Grinsell $\mathrm{D}$, Ashton MW, et al. Modern adjuncts and technologies in microsurgery: an historical and evidence-based review. Microsurgery 2010;30:657-66.

24. Pérez M, Sancho J, Ferrer C, García O, Barret JP. Management of flap venous congestion: The role of heparin local subcutaneous injection. Journal of Plastic, Reconstructive \& Aesthetic Surgery 2014;67:48e55e. 